\title{
Cultural Adaptation of the Extended Aberdeen Spine Pain Scale: A Turkish Version Study
}

\author{
Ilker ILHANLI' ${ }^{1}$, Necip GUDER ${ }^{2}$, Murat GUL ${ }^{3}$, Erhan ARSLAN ${ }^{4}$, Canan CELIK ${ }^{1}$ \\ ${ }^{1}$ Giresun University, School of Medicine, Department of Physical Medicine and Rehabilitation, Giresun, Turkey \\ ${ }^{2}$ Physical Medicine and Rehabilitation State Hospital, Giresun, Turkey \\ ${ }^{3}$ Giresun University, Faculty of Science, Department of Statistics, Giresun, Turkey \\ ${ }^{4}$ Giresun University, School of Medicine, Department of Neurosurgery, Giresun, Turkey
}

\section{ABSTRACT}

AIM: To evaluate the validity and reliability of Turkish version of Extended Aberdeen Spine Pain Scale (EASP).

MATERIAL and METHODS: After cultural adaptation, the questionnaire was administered to 120 patients. Test-retest reliability, internal consistency, construct and criterion validity and responsiveness to treatment were measured. All questionnaires were administered at baseline, 1 day later and at the end of treatment. Patients were assessed with Physical and Mental Component scores of SF-36 (SF-36PCS, SF-36MCS), Beck Depression Inventory (BDI) and Visual Analog Scale for pain (VAS).

RESULTS: Retest scores were significant (ICC: 0.878). For internal consistency, Cronbach's alpha was 0.908, which means all parts of the questionnaire are highly homogenous. For construct validity, Spearman's Correlation Coefficient was 0.907, which means that items of the questionnaire behaved as expected. For criterion validity of EASP, Spearman's rho correlations with SF36PCS (-0.999), SF-36MCS (-0.367), BDI (0.350), VASactivity (0.429), VASrest (0.399) and VASsleep (0.308) were found significant $(p=0.000)$. According to responsiveness, EASP, SF-36PCS, VASactivity, VASrest, VASsleep showed significant improvement after the treatment $(p=0.000)$.

CONCLUSION: The Turkish version of EASP seems reliable, valid, and responsive. Because of its usefulness for evaluating the whole spine as a functional unit, the EASP can be recommended for clinical trials.

KEYWORDS: Extended Aberdeen spine pain scale, Reliability, Validity

ABBREVIATIONS: EASP: Extended Aberdeen spine pain scale, SF-36 PCS: Physical component of SF-36, SF-36 MCS: Mental Component of SF-36, BDI: Beck depression inventory, VAS: Visual analog scale.

\section{INTRODUCTION}

$\mathrm{M}$ ost physicians agree on to treat the whole spine as a functional unit, but the higher incidence of low back pain (LBP) prevents interest in questionnaires evaluating the whole spine. Self-reported functional disability is accepted as the most valid outcome measure and there are several tools for assessing the $\operatorname{LBP}(5,7,13-15,17,18,20,21)$.
Standardized self-reported questionnaires allow analysis of symptoms, functions, responsiveness to treatment and quality of life. These questionnaires are generic, or domain- or disease-specific (9). Domain- and disease-specific instruments have the potential to be more responsive and sensitive than generic instruments (8), and their clinical importance for measuring treatment outcomes are highlighted (19). Due to the need for assessing the whole spine as a functional unit, 
a self-administered, domain-specific instrument was created in 2001 by extending the Aberdeen Low Back Pain Scale (18) and named the Extended Aberdeen Spine Pain Scale (EASP) (23). Cultural adaptation of the EASP in the Turkish population has not been conducted yet.

With or without adaptation studies, there are several tools translated to Turkish for assessing low back pain. However, there is no instrument evaluating the whole spine as a unit or adapted to the Turkish population. In this study, we aimed to evaluate the validity and reliability of the Turkish version of EASP and the response of this instrument to physical therapy.

\section{MATERIAL and METHODS}

\section{Questionnaires}

The EASP is a 30 -item questionnaire that assesses the health status of patients with spine pain across several dimensions, including pain, physical impairment, and functional disability. There are 9 questions for general assessment, 7 for the neck, 3 for the upper back, 6 for the lower back, 2 for the neck+upper back and 3 for the upper back+lower back. Responses to the questions are summed and converted to score percentages between 0 and 100, with 0 representing the least disabled and 100 the most severely disabled (23).

Short Form-36 (SF-36) is a generic outcome measure evaluating physical and mental function with the Physical Component Score (PCS) and Mental Component Score (MCS) (22). Validity of the Turkish version of SF-36 has been studied and it was found to be valid (12). A higher score indicates better health.

The Beck Depression Inventory (BDI) consists of 21 questions (2). The Turkish version of the BDI was shown to be valid and reliable (10). A higher score shows disrupted mental health.

\section{Translations and Cultural Adaptation}

The translation and cross-cultural adaptation of the original questionnaire were performed in accordance with recently published guidelines. At first, the index was translated from English to Turkish by three bilingual authors, whose native languages were Turkish. These translations were reviewed and discussed by two authors and a synthesis was formed. This version was translated back to English by two Englishspeaking linguistic scientists. A committee (medical doctors and linguistic scientists) checked the grammar of the index and compared it with the original questionnaire. After a careful review and cultural adaptation, the pre-final Turkish version of the questionnaire was provided.

Testing the pre-final version was the final stage of the process (face validity). The purpose of this stage was to determine the comprehensibility of this version and its capacity to assess the intended parameters. Ten patients with LBP and 10 healthy participants completed the pre-final Turkish version of EASP and they were interviewed to probe about their general comments on the difficulty of the questionnaire or understanding the text. All patients and healthy participants agreed on the questionnaire, and the pre-final Turkish version of EASP was therefore accepted as the final questionnaire.

\section{Patients and Assessments}

The Turkish version of EASP was administered to 120 native Turkish-speaking patients (40 males and 80 females) who presented with neck pain, upper back pain, lower back pain or a combination of these. In accordance with the requirements of ethical standards (Helsinki Declaration), their informed consents were obtained and the study was approved by the institution.

To assess the test- retest reliability of the Turkish version of EASP, all questionnaires were administered at admission and 1 day later. All assessments were repeated at the end of treatment. Treatment was applied 5 days per week for 3 weeks and applied to all regions the patient complained of. To perform construct validity and evaluate the response to treatment, patients were also assessed by SF-36 PCS and MCS scores, BDI score, and Visual Analog Scale for pain (VAS) at activity, at rest and at sleep. VAS (0 to 10) is used to assess the severity of pain with 10 indicating the worst pain.

\section{Statistical Analysis}

Statistical analysis was performed with SPSS 17.0. $\mathrm{P}<0.05$ was considered statistically significant.

\section{Reliability}

Two types of reliability (reproducibility and internal consistency) were evaluated for the Turkish version of EASP.

Test-retest reliability (reproducibility) measures stability over time, by administering the same test to the same subjects at two points in time. In this investigation, the questionnaires were administered at admission and 1 day later for retest reliability. 9 patients reported a change in their health status during the time-span until retest and were not included in the test-retest measurement. Correlation of the total scores between two administrations was measured with the intraclass correlation coefficient (ICC) for test-retest reliability $(n=111)$. ICCs can vary from 0.00 to 1.00 , where values of 0.60 to 0.80 are regarded as indicating good reliability and those above 0.80 as indicating excellent reliability (6).

Internal consistency measures how well all the questions in a scale are correlated with each other, and high interitem correlations may suggest that all the questions measure the factor of interest. Internal consistency is expressed by Cronbach's alpha, which is a measure of the reliability of the summative rating scale. Cronbach's alphas can range from 0 to 1.0 , where 1.0 indicates perfect internal consistency. Cronbach's alphas of greater than 0.80 in a scale are considered acceptable (3).

\section{Validity}

Construct validity is a major component in the testing of all outcomes instruments. It means that the scales in the questionnaire behave as expected.

Construct validity was measured by the Spearman correlation coefficient (SCC). The SCC was used because of the limited sample size and non-normal distributions. A value between 0 and 0.25 is accepted as 'no or poor', $0.26-0.50$ as 'moderate', 
$0.51-0.75$ as 'good', and $0.76-1.00$ as 'very good' correlation (4).

For testing the criterion validity, EASP scores were compared with the validated questionnaires (SF-36, BDI) and VAS by Spearman's rho correlation.

\section{Responsiveness (Sensitivity Towards Change)}

An outcome instrument of health status should detect the response to treatment; it should be responsive to significant clinical change. Responsiveness was measured by comparison of the each evaluation step by the Mann-Whitney $U$ test.

\section{RESULTS}

The Turkish version of EASP was administered to 120 native Turkish-speaking patients (40 males and 80 females) who were admitted with LBP. Nine patients reported a change in their health status during the time-span until retest and were not included test-retest measurement. Two patients did not attend the treatment. Seven patients did not complete all the questionnaires. After the exclusion of patients who could not complete the whole questionnaires or the treatment, statistical analysis was administered to 111 participants (38 males and 73 females). Mean age was $55.18 \pm 16.34$ years and the completion time was approximately 11 minutes for EASP. Thirteen patients $(11.7 \%)$ had only low back pain; 7 patients $(6,3 \%)$ had neck and low back pain; 5 patients $(4,5 \%)$ had upper back and lower back pain; 82 patients $(73.9 \%)$ had neck, upper back and lower back pain; 4 patients $(3,6 \%)$ had only neck pain. Duration of complaints was more than 6 months in all patients. Standard physical therapy including Transcutaneous Electrical Nerve Stimulation, Ultrasound, Hotpacks, Exercise, and medication with acetaminophen or non-steroid anti-inflammatory drugs was administered to all patients.

\section{Reliability}

For test-retest reliability, a total of 111 patients who did not report changes in health status according to the transition question in both the baseline and the retest questionnaire.
Retest scores were significant and high for EASP (ICC:0.878, $\mathrm{n}=111$ ). When test-retest reliability was evaluated for low back $(n=13)$, for neck+lower back $(n=7)$, for upper back+lower back $(n=5)$, for neck+upper back+lower back $(n=82)$, and for the neck $(n=4)$ separately, retest scores were $0.788,0.867,0.918$, 0.855 , and 0.123 , respectively (Table I).

For internal consistency of EASP, Cronbach's alpha of the final questionnaire was 0.908 , which means all parts of the questionnaire were highly homogenous $(n=111)$. Cronbach's alpha for each region was $0.792,0.890,0.924,0.902,0.963$, respectively (Table I).

\section{Validity}

For construct validity of EASP, SCC of the final questionnaire was 0.907 , which means that the scales in the questionnaire behaved as expected $(n=111)$. SCC of each region was calculated as $0.750,0.877,0.914,0.902,0.923$, respectively (Table I).

For criterion validity of EASP, Spearman's rho correlations with SF-36 PCS (-0.999, $p=0.000)$, SF-36 MCS $(-0.367, p=0.000)$, BDI $(0.350, p=0.000)$, VASactivity $(0.429, p=0.000)$, VASrest $(0.399, p=0.000)$ and VASsleep $(0.308, p=0.000)$ were found to be significant and acceptable. Correlations between SF-36 PCS and other variables were similar to correlations of EASP (Table II). Correlations between SF-36 MCS and VASactivity, VASrest, VASsleep were significant but poor $(-0.096 p=0.043$; $-0.166 p=0.000 ;-0.118 p=0.013$; respectively). While only the correlation between BDI and VASrest was significant $(0.134$ $\mathrm{p}=0.005)$, correlations between BDI and VASactivity, VASsleep were not significant $(\mathrm{p}>0.05$, Table II).

\section{Responsiveness}

Means \pm standard deviations of all variables for each evaluation step are shown in Table III. According to responsiveness; EASP, SF-36 PCS, VASactivity, VASrest, VASsleep showed significant improvement after the treatment $(p=0.000$ for each, Table IV). SF-36 MCS and BDI did not show significant difference by treatment ( $p>0.05$, Table IV).

Table I: Intraclass Correlation Coefficient for Test-Retest Reliability, Cronbach's Alpha for Internal Consistency and Spearman Correlation Coefficient for Construct Validity

\begin{tabular}{llcl}
\hline & ICC & Cronbach's Alpha & sCC \\
\hline EASP $(n=111)$ & 0.878 & 0.908 & 0.907 \\
\hline Lower back $(n=13)$ & 0.788 & 0.792 & 0.760 \\
\hline Neck+Lower back $(n=7)$ & 0.867 & 0.890 & 0.877 \\
\hline Upper back+Lower back $(n=5)$ & 0.918 & 0.924 & 0.914 \\
\hline Neck+Upper back+Lower back $(n=82)$ & 0.855 & 0.902 & 0.902 \\
\hline Neck $(n=4)$ & 0.123 & 0.963 & 0.923 \\
\hline
\end{tabular}

ICC: Intraclass correlation coefficient, 1.0 indicates perfect correlation, Cronbach's alpha: 1.0 indicates perfect internal consistency, SCC: Spearman correlation coefficient, 0.51-0.75 indicates 'good', 0.76-1.00 indicates 'very good' correlations, EASP: Extended Aberdeen spine pain scale. 
Table II: Spearman's Rho Correlations for Testing Criterion Validity $(n=111)$

\begin{tabular}{|c|c|c|c|c|c|c|}
\hline & EASP & SF-36 PCS & SF-36 MCS & BDI & VAS activity & VAS rest \\
\hline SF-36 PCS & $-0.999^{\star \star}$ & 1.000 & & & & \\
\hline SF-36 MCS & $-0.367^{\star \star}$ & $0.364^{\star \star}$ & 1.000 & & & \\
\hline $\mathrm{BDI}$ & $0.350^{\star *}$ & $-0.348^{\star \star}$ & $-0.975^{\star \star}$ & 1.000 & & \\
\hline VAS activity & $0.429^{\star *}$ & $-0.427^{\star *}$ & $-0.096^{\star}$ & 0.060 & 1.000 & \\
\hline VAS rest & $0.399^{\star \star}$ & $-0.395^{\star \star}$ & $-0.166^{\star \star}$ & $0.134^{\star *}$ & $0.555^{\star \star}$ & 1.000 \\
\hline VAS sleep & $0.308^{\star *}$ & $-0.306^{\star \star}$ & $-0.118^{*}$ & 0.082 & $0.414^{\star *}$ & $0.644^{\star *}$ \\
\hline
\end{tabular}

*Significance level: $p<0.05,{ }^{* *}$ Significance level: $p<0.01$, EASP: Extended Aberdeen spine pain scale, SF-36 PCS: Physical component of short form 36, SF-36 MCS: Mental component of short form 36, BDI: Beck depression inventory, VAS: Visual analog scale.

Table III: Means of Variables for Each Evaluation Step (Means $\pm S D, n=111$ )

\begin{tabular}{lccccccc}
\hline Evaluation step & EASP & SF-36 PCS & SF-36 MCS & BDI & VAS activity & VAS rest & VAS sleep \\
\hline Baseline & $48.97 \pm 18.87$ & $38.70 \pm 10.15$ & $58.87 \pm 5.27$ & $10.48 \pm 5.41$ & $5.28 \pm 2.14$ & $3.57 \pm 2.53$ & $3.56 \pm 2.65$ \\
\hline Retest & $48.98 \pm 18.86$ & $38.69 \pm 10.15$ & $58.92 \pm 5.27$ & $10.45 \pm 5.40$ & $5.27 \pm 2.14$ & $3.57 \pm 2.53$ & $3.54 \pm 2.67$ \\
\hline End of treatment & $34.14 \pm 19.37$ & $47.31 \pm 11.03$ & $59.13 \pm 5.17$ & $10.41 \pm 5.37$ & $2.87 \pm 1.83$ & $1.45 \pm 1.63$ & $1.35 \pm 1.65$ \\
\hline
\end{tabular}

SD: Standard deviation, EASP: Extended Aberdeen spine pain scale, SF-36 PCS: Physical component of short form 36 , SF-36 MCS: Mental component of short form 36, BDI: Beck depression inventory, VAS: Visual analog scale.

Table IV: Comparison of Evaluation Steps for Each Variable to Evaluate Responsiveness $(n=111)$

\begin{tabular}{lccccccc}
\hline $\begin{array}{l}\text { Comparison of } \\
\text { Evaluation steps }\end{array}$ & $\begin{array}{c}\text { EASP } \\
\text { p value }\end{array}$ & $\begin{array}{c}\text { SF-36 PCS } \\
\text { p value }\end{array}$ & $\begin{array}{c}\text { SF-36 MCS } \\
\text { p value }\end{array}$ & $\begin{array}{c}\text { BDI } \\
\text { p value }\end{array}$ & $\begin{array}{c}\text { VAS activity } \\
\text { p value }\end{array}$ & $\begin{array}{c}\text { VAS rest } \\
\text { p value }\end{array}$ & $\begin{array}{c}\text { VAS sleep } \\
\text { p value }\end{array}$ \\
\hline $1-2$ & 0.997 & 0.996 & 0.921 & 0.957 & 0.929 & 1.000 & 0.943 \\
\hline $1-3$ & $0.000^{*}$ & $0.000^{*}$ & 0.438 & 0.919 & $0.000^{*}$ & $0.000^{*}$ & $0.000^{*}$ \\
\hline $2-3$ & $0.000^{*}$ & $0.000^{*}$ & 0.524 & 0.966 & $0.000^{*}$ & $0.000^{*}$ & $0.000^{*}$ \\
\hline
\end{tabular}

*Significance level: $p<0,05$, 1-2: Baseline versus retest, 1-3: Baseline versus end of treatment, 2-3: Retest versus end of treatment, EASP: Extended Aberdeen spine pain scale, SF-36 PCS: Physical component of short form 36, SF-36 MCS: Mental component of short form 36, BDI: Beck depression inventory, VAS: Visual analog scale.

\section{DISCUSSION}

The aim of the present study was to evaluate the validity and reliability of Turkish version of EASP and evaluate the response of this instrument to physical therapy. The quality of a measurement is based on reliability, validity, and ease of use (11). The EASP was adapted to the Turkish population using recommended guidelines (1). We did not consider the details of treatment modalities or the type of disorders in our study, because the aim of this study was to evaluate the response of EASP to changes due to treatment. These treatment regimens could be diversified from physical therapy to surgical interventions. This was a single-center study with patients suffering from neck pain, upper back pain, lower back pain or a combination of these. The completion time was approximately 11 minutes, and patients indicated that it was an acceptable length, but this time was a little longer than in the literature (23). Patients had no difficulty in completing the questionnaire. The ease of administration allowed the EASP to be easily given in a clinical setting.
The drop out number of the study was approximately $7.5 \%$ of the total subject number. Although the sample size was adequate for instrument testing and cultural adaptation, the number of subjects for each region should be increased. The number of subjects was low for all regions other than the patients suffering from the whole spine $(n=82)$. There was no patient with upper back pain alone. Probably, low ICC $(0.123)$ for the neck was due to the low subject number $(n=4)$. When the subject number was low, a little difference between the answers of baseline and retest can cause a high decrease of ICC. However, we should consider the test-retest score for the whole spine (ICC:0.878), because our aim was to evaluate the whole spine as a unit. The test-retest score for EASP was high and acceptable, which indicated the reproducibility of this instrument. This was similar to that found with other studies $(16,23)$. Realism of the retest coefficients may seem questionable because the retest questionnaire was administered after only 1 day. However, we consider it unlikely that the patients remember their answers given 1 day before, 
particularly as there are more than one questionnaires and many items that have to be kept in mind. If there was a longer time-span, it might also have caused a bias because of a change in health status by the ongoing therapy. Nine patients in our study reported a change in health status before retest and they were excluded from test-retest analysis.

In our study, Cronbach's alpha was 0.908 and EASP was found to have high internal consistency, similar to other studies $(16,23)$. Also, Cronbach's alphas of each region were high and acceptable, too. This indicates that the Turkish version of EASP has high internal consistency for all items of the questionnaire, which means all parts of the questionnaire are highly homogenous.

For construct validity, Spearman's correlation coefficient (SCC) was 0.907 , which means that the items in the questionnaire behaved as expected. For example, the scores got better with improved health status or got worse with deteriorating health status. Also, the SCC of each region was more than 0.76 and these were also acceptable. The construct validity of the questionnaire reported in previous studies was similar to our findings $(16,23)$.

Similar to the literature, significant correlations were found between EASP and other measures $(16,23)$. High correlations between EASP and the generally accepted measure SF-36 PCS suggest that both questionnaires actually measure health status affected by spine pain. Relatively low but significant correlations between EASP and other measures were similar to the correlations between SF-36 PCS and other measures. Also, as seen in Table III and IV, all scales showed a numerically and statistically significant improvement with treatment, except the scales for mental health status (SF-36 MCS and BDI). Probably, this was due to better or stable mental health status of the subjects in our study. Nonetheless, Table IV shows the responsiveness of EASP to treatment and EASP can be used for evaluating the efficacy of a treatment regimen.

\section{- CONCLUSION}

The Turkish version of the EASP seems to be reliable, valid, and responsive. Furthermore, because of its usefulness for evaluating the whole spine as a functional unit, the EASP can be recommended for clinical trials investigating the efficacy of therapeutic regimens for the spine.

\section{REFERENCES}

1. Beaton DE, Bombardier C, Guillemin F, Ferraz MB: Guidelines for the process of cross-cultural adaptation of self report measures. Spine 25: 3186-3191, 2000

2. Beck AT: An inventory for measuring depression. Arch Gen Psychiatry 4: 561-571, 1961

3. Bellamy N: Musculoskeletal Clinical Metrology. Boston: Klumer Academic, 1993

4. Dogan SK, Ay S, Evcik D, Baser O: Adaptation of Turkish version of the questionnaire Quick Disability of the Arm, Shoulder, and Hand (Quick DASH) in patients with carpal tunnel syndrome. Clin Rheumatol 30: 185-191, 2011
5. Fairbank JC, Couper J, Davies JB, O'Brien JP: The Oswestry low back pain disability questionnaire. Physiotherapy 66: 271-273, 1980

6. Feinstein AR: Clinimetrics. New Haven: Yale University Pres, 1987

7. Greenough CG, Fraser RD: Assessment of outcome in patients with low back pain. Spine 17: 36-41, 1992

8. Guyatt $\mathrm{GH}$ : A taxonomy of health status instruments. J Rheumatol 22: 1188-1190, 1995

9. Guyatt GH, Feeny DH, Patrick DL: Measuring health-related quality of life. Ann Intern Med 118: 622-629, 1993

10. Hisli N: The reliability and validity study of the Beck Depression Inventory in a Turkish sample. Psikoloji Dergisi 6:118-122, 1988 (In Turkish)

11. Hobby JL, Watts C, Elliot D: Validity and responsiveness of the patient evaluation measure as an outcome measure for carpal tunnel syndrome. J Hand Surg 30: 350-354, 2005

12. Kocyigit $H$, Aydemir O, Fisek G, Olmez N, Memis A: Kısa form36 'nın Türkçe versiyonunun güvenilirliği ve geçerliliği. İlaç ve Tedavi 12(2): 102-106, 1999 (In Turkish)

13. Kopec JA, Esdaile JM, Abrahamowicz M, Abenhaim L, WoodDauphinee S, Lamping DL, Williams JI: The Quebec back pain disability scale: Measurement properties. Spine 20: 341-352, 1995

14. Lawlis GF, Cuencas R, Selby D, McCoy CE: The development of the Dallas pain questionnaire. An assessment of the impact of spinal pain on behaviour. Spine 14: 510-516, 1989

15. Million R, Hall W, Nilsen $\mathrm{KH}$, Baker RD, Jayson MIV: Assessment of the progress of the back pain patient. Spine 7: 204-212, 1982

16. Osthus H, Cziske R, Jacobi E: A German version of the extended Aberdeen back pain scale. Spine 31: 571-577, 2006

17. Roland M, Morris R: A study of the natural history of back pain. Part 1: development of a reliable and sensitive measure of disability in low back pain. Spine 8: 141-143, 1983

18. Ruta DA, Garratt AM, Wardlaw D, Russell IT: Developing a valid and reliable measure of health outcome for patients with low back pain. Spine 19: 1887-1896, 1994

19. Stucki G, Daltroy L, Liang MH, Lipson SJ, Fossel AH, Katz $\mathrm{JN}$ : Measurement properties of a self-administered outcome measure in lumbar spinal stenosis. Spine 21: 796-803, 1996

20. Tesio L, Granger CV, Fiedler RC: A unidimensional pain/ disability measure for low back pain syndromes. Pain 69: 269-278, 1997

21. Wadell G, Main CJ: Assessment of severity in low back disorders. Spine 9: 204-208, 1984

22. Ware JE, Sherbourne CD: The SF-36 health status survey: 1. Conceptual framework and item selection. Med Care 30: 473-483, 1992

23. Williams NH, Wilkinson C, Russell IT: Extending the Aberdeen Back Pain Scale to include the whole spine: A set of outcome measures for the neck, upper and lower back. Pain 94: 261274,2001 